\title{
The roadmap towards a holistic safety culture based on an integrated environmental health and safety solution landscape
}

\author{
B. Freibott \\ SAP Germany $A G \&$ Co. KG, Germany
}

\begin{abstract}
Environmental health and safety (EHS) is too often considered as a functional area or as a center of specific expertise and EHS managers are seeing themselves too often and too much as a capsulated competence center. Such an approach to EHS will always fall far short of what is really needed for a successful, holistic, proactive and preventive safety culture. EHS is in fact an environment or network of many stakeholders with very different rolls, objectives, expertise and backgrounds. And it is a cobweb of many processes and functions within and outside of EHS, which are all correlated with dependencies and interdependencies. Above all: EHS is not an island within a corporation, but part of the production chain and part of the operative process, from sustainable purchasing and supply change, to safe operations, sustainable product design and productions, and on to market access and transportation. And within the network of markets, customers, competitors, regulators, NGOs, and investors it is more and more a crucial and inevitable prerequisite to assure save and sustainable operations based on a sustainability strategy, and to measure sustainability performance and make the sustainability achievements visible. This can only be achieved in a holistic concept or strategy that considers the classical enterprise resource planning (ERP) in the same way as it considers the broad landscape of functions and processes within EHS, i.e. assuring safety and the protection of co-workers health, facilitate safe operation, minimize the environmental impact of the operation, and design and produce sustainable, marketable products, and by this way to drive brand safety and the triple bottom line of profit, social performance and environmental performance.
\end{abstract}

Keywords: EHS management system, incident management, incident prevention, information technology (IT), operational excellence. 


\section{Introduction}

Analyzing the status quo of today's company, one comes to a rather heterogeneous picture. There are companies - too many companies - that still are focusing on compliance, i.e. on doing what is necessary to comply with regulations. Their approach is rather reactive, i.e. reacting on regulation or reacting on incidents and mishaps.

There is a second group of companies that have established a very organized and systematic approach to safety. The objective is to prevent incidents from happening by use of safety management systems, methods and regulations. Their objective is to create a 'reasonably' safe environment, based on legislation and best practice, however, incidents are very often still considered a natural consequence of the operation, i.e. incidents are bound to happen every now and then.

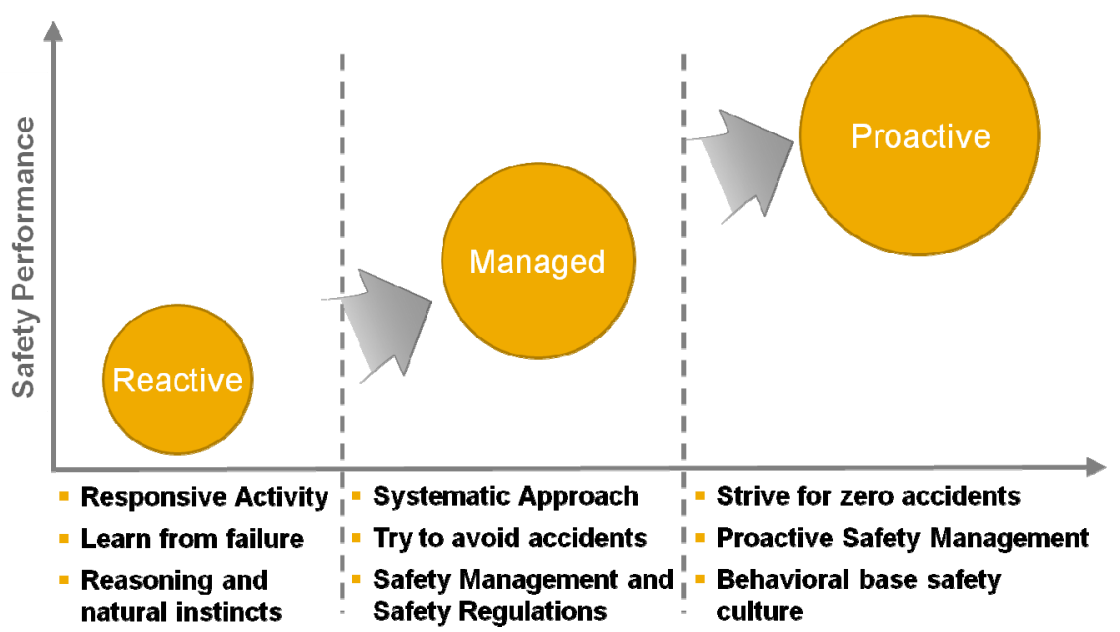

Figure 1: Safety culture maturity levels.

The third and most mature group of companies is striving for a zero incident environment. The essential objective is: It is not acceptable that the employees are put on any kind of avoidable danger and risk, or that the operation is posing any risk for the environment. These companies base their approach on a broad approach involving everybody into a corporate safety culture. This approach implies that really everyone in a corporation is part of this safety culture and that all corporate functions are sharing this safety culture objective. Operational safety - or better operational excellence - has to be a corporate objective intertwining with all operational areas. In such an environment of preventive safety culture, safety management cannot be handled as an isolated functional area by capsulated experts that focus on safety processes and regulations to drive compliance and best practices only. 
Such an approach requires

- a comprehensive integrated approach,

- connecting people and functions that are normally maybe not even connected or interacting,

- $\quad$ integrating data from various functional areas for a holistic picture and to have a sound database from which to derive actions and activities,

- creating the ability to monitor operational excellence and safety performance on a near to real-time level,

to establish a corporate safety culture and operational excellence model, to monitor this environment at any moment, and to react in due time on any alteration within the organization.

To achieve this level of operational excellence, most companies - as mentioned before - have still a long way ahead, i.e. it is time to think about the long-term operational excellence aspirations and about the way forward, the roadmap to a successful safety culture.

Especially when a company is coming from a rather rudimentary safety organization with a rather reactive approach to safety management, it is important to have the necessary insight into actual performance to drive the evolution, to become more systematic with a managed approach, and in the final transformation phase, to create - based on experience and insight - a practiced safety culture and proactive safety management.

The right approach can only go via a clear picture of the ideal world, i.e. the final scenario, from which a suitable and very individual roadmap has to be derived. It starts from the status quo with all its restrictions and limitations, to develop the roadmap, and to implement step by step the system prerequisites, policies, processes, etc. and - above all - to drive development in the relevant people.

\section{Change process outlines}

Anybody who faces the challenge of implementing a comprehensive setup for a proactive safety management system to support operational excellence as mentioned above, including all the various aspects and facets of such a corporate concept, will need a systematic approach or road map for the way forward.

Such a systematic approach can have different designs or granularities, but roughly there are five principal steps to keep in mind:

1. Problem analysis

2. Process design

3. Integration design

4. Solution setup

5. Flexible deployment 


\section{Problem analysis:}

First of all, perform a detailed and concise analysis of the status quo, of the present situation, of the challenges, and of the objectives that have been set for a new corporate concept for operational excellence.

\section{Process design:}

Based on step one and focusing on the future vision for the operational excellence concept, a detailed design should be done. Such a design should not focus on functions but rather on processes - processes that in many cases are cross functional and interdisciplinary. In case of safety management, this becomes apparent when drawing up the flows of action and information across the organization and over organizational boundaries.

\section{Integration design:}

Very quickly it also becomes visible where information is needed from which area - that is, where there are integration needs between the functional areas. The previously discussed comprehensiveness of a corporate operational safety approach inevitably creates a level of complexity of an EHS solution landscape with many facets of dependencies, interdependencies and a high degree of collaboration. Consequently, system interaction is an important factor for success.

\section{EHS solution setup:}

The next step is the setup of a system or of processes to facilitate the operational excellence and safety activity. Given the many very different stakeholder groups around EHS and operational excellence and their very different needs, it is obvious that to be suitable and acceptable a solution must have a certain degree of user orientation, adaptability, and ease of use. Apart from the pure need for the processes, the usability of the solution and its acceptance by the users are key factors for success.

\section{Flexible Deployment:}

Last, but still of great importance, is the running of this environment of operational excellence, which should be driven as a flexible deployment. A key factor of success for organizations today is to keep pace and sustain momentum as a learning organization.

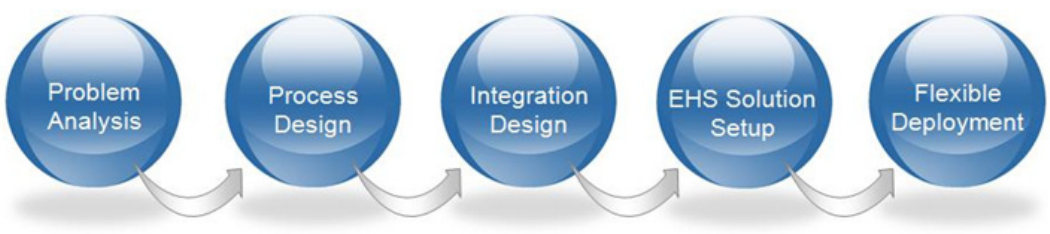

Figure 2: Phases of deployment. 


\section{The comprehensive setup - bringing safety into shape}

As we have seen, it is necessary to achieve a systematic and comprehensive approach for a proactive and successful safety culture. This means to bring together all stakeholders into a complete picture, but also to leave in this picture enough flexibly to act and react within a highly volatile organizational environment.

Starting from the status quo, it has to be analyzed where are the various links between both the functional areas within EHS and between EHS and other units of the corporation, as shown in the diagram below.

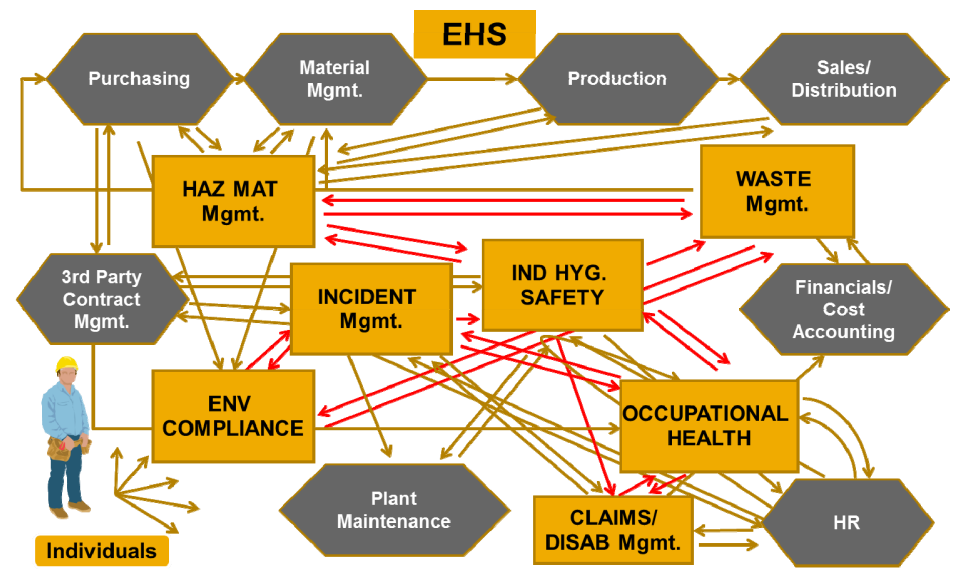

Figure 3: EHS - a complex reality!

This picture shows only a part of the world around EHS, but it already makes clear that one is facing a complex reality.

First of all, there is the chain of links between Hazardous Substance Management (HSM), Industrial Hygiene (IH) and Occupational Health $(\mathrm{OH})$. HSM deals with the materials that constitute a certain element of risk for the company, employees and environment. The same materials become an integral part of the exposure profiles in IH as soon as people are exposed to them. The exposure profiles within Health Risk Assessment (HRA) and information from Human Resources (HR) together form the basis for medical protocol planning in $\mathrm{OH}$. There is also a clear link between Waste Management and HSM, since hazardous substances very often end up as waste, and this waste often has to be handled as a special form of hazardous substance. As such, it again constitutes an exposure for co-workers involved in waste handling. Moreover, it is important to state that HSM is a function that is closely intertwined with the material flow within a company from the outset - from the point of purchase until the substance leaves the company as a product, part of a product or waste. Waste Management is also connected to purchasing and Material Management to a certain extent in terms of the procurement of waste disposal services and the 
storage of waste. Furthermore, the financial dimension of waste disposal is also of crucial importance, since waste management costs contribute to overall material costs and may in fact influence purchasing decisions with respect to alternative substances. As a result, it is necessary to link this component with Cost Accounting and Financial Accounting. Waste Management also incorporates EC (Environmental Compliance), i.e. legal reporting with respect to appropriate waste disposal.

The functional area of Incident and/or Accident Management with Hazard Reporting/Near-miss Reporting incorporates a range of integration aspects. The aftermath of an incident often brings numerous other functional areas into play, including Claims Management, Disability Management and Return-to-Work. Injuries and subsequent medical treatment necessitate the involvement of $\mathrm{OH}$, e.g. information about the injuries and first-aid treatments that are needed as input for incident reporting. Of particular importance is the link between Incident Management and IH as a principal trigger of incident prevention, since the incidents, hazard reports and near-miss reports are a key starting point for corrective actions measures designed to make work areas safer. There is also an unavoidable link between the areas of Disability Management, HR and $\mathrm{OH}$. The reintegration strategies for co-workers with restrictions are compiled in cooperation with reintegration managers, $\mathrm{HR}$ and $\mathrm{OH}$ along with additional input from IH, e.g. information about exposures in alternative work areas. HR itself is interlinked with many functional areas in EHS related to the workforce, i.e. any area that deals with safeguarding the employees. Parallel to this HR integration, there is also a link to third-party management, since whatever applies to in-house employees also applies to external employees such as contractors. It is also possible to draw a direct link between $\mathrm{IH}$ and Plant Maintenance as regards the corrective measures that have to be triggered as a follow-up to HRA and incidents. When discussing incidents, it is impossible to ignore Incident Management for Environmental Compliance Management. This is particularly true during a leakage, outlet or spill that is harmful to the environment and subject to legal reporting obligations. With regard to regular emissions such as greenhouse gases, $\mathrm{CO}_{2}$ or other kinds of emissions subject to legal reporting, the necessary consumption information with regard to input quantities needs to be obtained from purchasing or material management in order that the relevant emission figures can be calculated.

Furthermore, it is crucial to mention one particular interface, the importance of which cannot be overstated: The human interface. In any holistic EHS approach there is the 'individual' to be considered as a key factor for the success of an EHS Management System. Alongside expert users such as industrial hygienists, occupational health practitioners, etc., there are many other stakeholders, who need to be involved in the concepts for EHS. There are various hierarchies of management, each with very different information requirements ranging from very condensed and exception-triggered C-level reporting down to the manager's view of his team members exposures, $\mathrm{OH}$ planning, safety training, and so on. 
Finally, of very particular importance, one has to consider all employees and contractors as a whole, i.e. everybody who is active in the company. It is a generally accepted fact that the top level of safety awareness can only be achieved by incorporating everybody into the EHS concept. In other words, everybody has to be a part of the EHS world. This can be achieved uniquely by involving all individuals, e.g. via self-service portals for EHS, employee info centers with information about exposures, safety regulations, PPE (personal protective equipment), planned and executed medical protocols, and so on. Transparency and inclusion is the first and most important step towards achieving an excellent behavioral safety culture. And to deliver a successful holistic EHS approach, it is essential to make the integration of people a core element of the EHS strategy from the very outset.

If we also take aspects of Product Safety/Product Compliance into account in such a concept, we would very likely discover even more interdependencies and interactions that would need to be added to the solution landscape setup for EHS. Another aspect that further compounds this complexity is that most modern companies are part of a global economic system. Their customers, partners and contractors are international. Similarly, most industries are interwoven into a global system of plants and subsidiaries in various countries and jurisdictions with differences in terms of language and culture. When seeking to design a successful global corporate EHS strategy that will work for all regions and adequately support all languages and local or regional requirements, it is imperative that this international or global aspect is given sufficient consideration.

Essentially, all these aspects of interaction and interdependence point towards one crucial message - an isolated approach to any functional area of EHS is bound to miss the target.

If we bring such a comprehensive approach to EHS now into the broader complex of operational excellence, this means to intertwine it further with the world that what we might call Enterprise Asset Management (EAM).

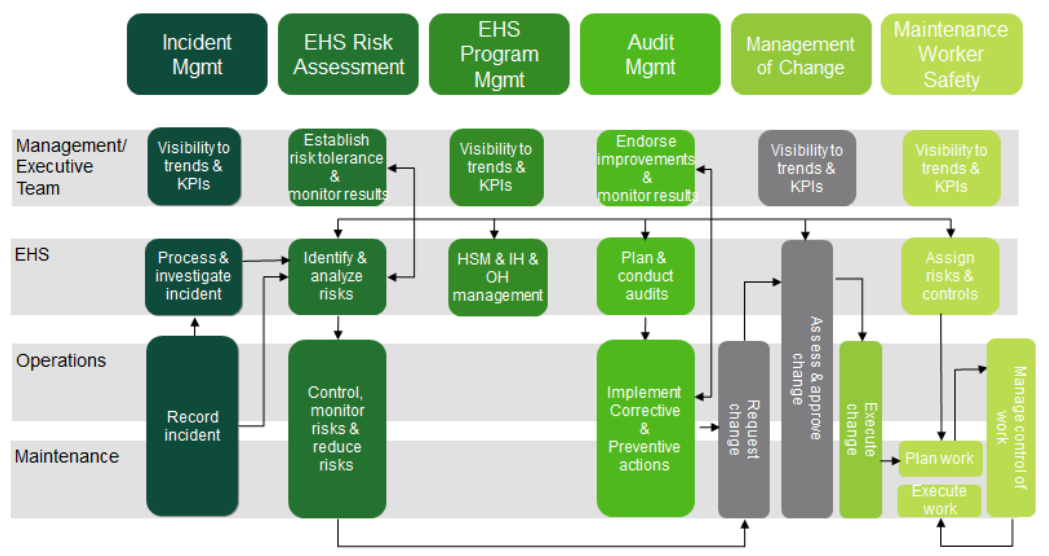

Figure 4: Functional map for operational safety. 
Bringing these worlds then closer together, one quickly realizes that they are not only info exchanges or interfaces. There is a great quantity of overlap of functional areas and of processes.

Just for an example: A hazard or potential danger is discovered. This hazard is reported in incident as hazard observation, which, as natural consequence triggers audits, risk assessments, preventive measures, corrective actions etc. The health risk assessment might detect some particular exposure that makes it necessary to execute some change in the process, in the flow of production, in the material composition or something else. The change itself constitutes a new and particular risk. It is a well-known fact that many incidents and accidents happen during irregular activity, like maintenance and changes in assets and operations. Consequently, it might be decided to make this change subject to a Management-of-Change $(\mathrm{MoC})$ process. Within $\mathrm{MoC}$ there are established standard operation procedures for change processes with special steps for example to assure the safe execution of changes. Examples for this are pre-change risk assessment, post-change risk assessments, pre-start-up safety procedures, post startup risk assessments etc. Within this complexity, the change itself is only one step, embedded in all relevant procedures to assure safe operation at any point of time. The change itself, e.g. maintenance activity, is then again subject to specific change-related safety procedures, like shut-down and tag-out procedures. The maintenance workers have to be aware of all latent dangers and exposure, they have to be informed about all relevant safety measures, and depending on the exposure and activity they might need special training or medical checkups related to the intervention that is about to be executed.

In simple words: There is a whole complex chain of processes and activity that can be triggered from various points within the operation, between maintenance and safety management, and - not least - at any point of time there has to be certain level of transparence for operations management to enable management to comply with the necessary standards of care and diligence.

No stake-holder and no activity can be considered as an isolated task or responsibility. Everybody and all processes have to be part of a complex, highly integrated and flexible system environment to assure what was previously defined as operational excellence.

\section{Conclusion}

We have seen so far, that safety management cannot be seen any more as a bundle of functional areas in a rather capsulated approach. On the way towards a proactive and preventive safety culture, more and more enterprises are implementing safety management and EHS as part of a comprehensive concept of operational excellence to deal with the challenges and risks. 


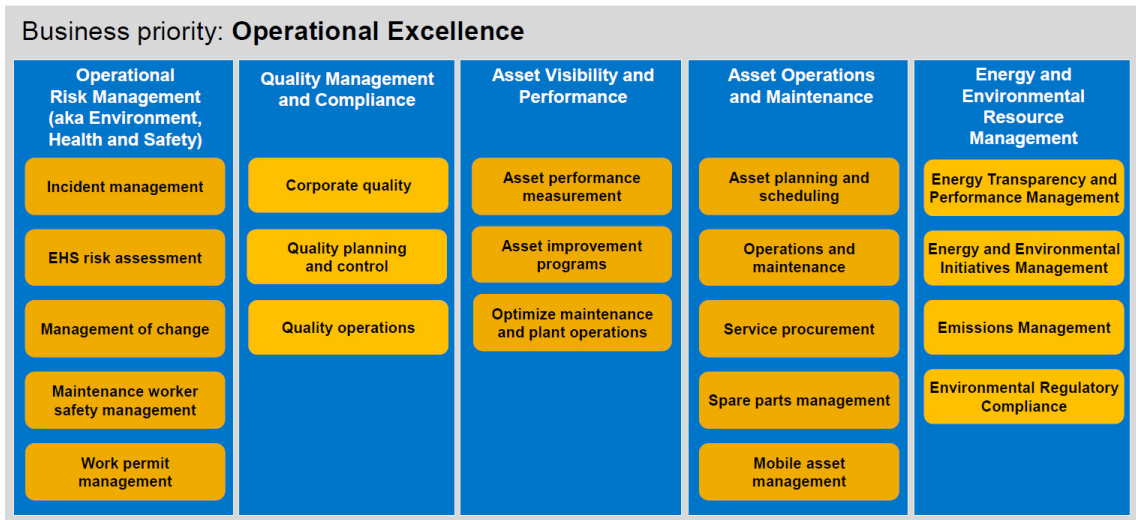

Figure 5: Business priority - operational excellence.

This implies:

- Asset Integrity - Assuring operational performance and maintenance with a high degree of visibility.

- Operational Risk Management - Detection, assessment and mitigation of all relevant risks of the operation, i.e. risks for the individuals, for the environment and for the enterprise.

- Energy and Resource Efficiency - Transparency of consumption with the object of optimization of consumption of natural resource to minimize the environmental footprint, to comply with environmental regulations and to raise efficiency and profitability of the operation.

- Quality Management - Assuring quality of materials and products to comply with regulations and guarantee marketability of the produce.

None of these entrepreneurial objectives can be achieved at a high level without keeping in mind the other areas of responsibility. Operational excellence and safe operation is not a functional area, but rather a kind of neuronal network of nodes and synapses across an operation. It is by its nature equally flexible and agile as it is fragile as any neuronal network. This brings us back to the prime question: What is safety? Safety is difficult to define, and safety can only be defined by the absence of something else, i.e. the absence of dangers and hazards. And safety can be assured only in a continual system of responsibility and alertness, without any danger of relapses into complacency. In other words: Safety is a continually maintained non-status which requires everybody at any point of time to be kept up! And the underlying safety management system has to reflect this network of activities and responsibilities, and facilitate it to bring it to a maximum efficiency.

\section{Nomenclature:}

$\begin{array}{ll}\text { EHS } & \text { Environment Health and Safety } \\ N G O & \text { Non-Governmental Organizations } \\ \text { ERP } & \text { Enterprise Resource Planning }\end{array}$


286 Risk Analysis IX

$\begin{array}{ll}\text { HSM } & \text { Hazardous Substance Management } \\ \text { IH } & \text { Industrial Hygiene } \\ \text { OH } & \text { Occupational Health } \\ \text { HR } & \text { Human Resources } \\ \text { HAZ MAT Mgmt. Hazardous Material Management } \\ \text { ENV Compliance (EC) Environmental Compliance } \\ \text { HRA } & \text { Health Risk Asset Management } \\ \text { PPE } & \text { personal protective equipment } \\ \text { EAM } & \text { Enterprise Asset Management } \\ M o C & \text { Management-of-Change } \\ I T & \text { Information Technology }\end{array}$

